\title{
La transition chinoise : pouvoir d'État et gestion de la société
}

Entretien conduit par Jean-Luc Racine

Jean-Luc Domenach et François Godement

\section{(2) OpenEdition}

\section{Journals}

Édition électronique

URL : http://journals.openedition.org/transcontinentales/553

ISBN : 978-2-8218-1112-6

ISSN : 1775-397X

\section{Éditeur}

Editions de la maison des sciences de l'homme

Édition imprimée

Date de publication : 31 décembre 2006

Pagination : 87-102

ISBN : 978-2-200-92170-5

ISSN : 1950-1684

\section{Référence électronique}

Jean-Luc Domenach et François Godement, « La transition chinoise : pouvoir d'État et gestion de la société », Transcontinentales [En ligne], 3 | 2006, document 5, mis en ligne le 12 octobre 2011, consulté le 07 septembre 2020. URL : http://journals.openedition.org/transcontinentales/553

\section{Ce document a été généré automatiquement le 7 septembre 2020}

Tous droits réservés 


\title{
La transition chinoise : pouvoir d'État et gestion de la société
}

\author{
Entretien conduit par Jean-Luc Racine \\ Jean-Luc Domenach et François Godement
}

\section{NOTE DE L'ÉDITEUR}

Jean-Luc Domenach est directeur de recherche à la Fondation nationale des sciences politiques, et directeur de l'Antenne franco-chinoise de sciences sociales et humaines de Pékin. François Godement est professeur à l'Institut d'études politiques de Paris, et président d'Asia Centre, Centre études Asie. Tous deux comptent parmi les meilleurs spécialistes de la Chine contemporaine.

Du débat qui les réunit autour de Transcontinentales, de multiples constats et de grandes interrogations sont tirés. La libéralisation de l'économie ne conduit pas au dépérissement de l'État, mais bien à sa consolidation technocratique. Le modèle de croissance débridée touche aujourd'hui à ses limites, car ses coûts sociaux et environnementaux deviennent trop lourds. Le Parti, qui tient l'État, doit donc gérer les crises naissantes. Il choisit d'imaginer des ajustements et de laisser des marges de manœuvre, tout en verrouillant l'expression des mécontentements. Ce pragmatisme suffira-t-il face à des défis structurels, démographiques et économiques? Si le régime ne tient que par l'espoir en l'avenir que suscite une extraordinaire croissance économique, le pouvoir saura-t-il gérer la croissance tout en limitant les effets négatifs de la surchauffe? Ou d'autres équilibres seront-ils trouvés, entre pouvoir et société ?

1 Transcontinentales : Voici un quart de siècle que les réformes économiques ont été engagées en Chine. Quel point peut-on faire sur la ligne retenue alors par Deng Xiaoping, et comment évaluer l'impact de cette réorientation sur la société chinoise et sur le pouvoir d'État? Pour le dire autrement, quel sens faut-il donner à une formule telle que « socialisme de marché »? Comment définir le -système actuel? 
2 François Godement : Je dirais qu'il y a miracle et continuité. Voici soixante-dix ans, l'essentiel des exportations chinoises était assuré par des entreprises étrangères. C'est de nouveau le cas aujourd'hui. Mais à l'époque, l'industrie chinoise représentait $1 \% \mathrm{du}$ PIB du pays, alors que désormais le seul commerce extérieur représente $60 \%$ du PIB, et ce à partir des exportations industrielles plutôt que des matières premières ou des services : c'est une véritable hypertrophie. Pour autant, comparer l'économie chinoise, pour une part cellulaire et fermée, pour une part ouverte et même dépendante, à l'économie-monde n'est pas nouveau. On s'interroge depuis un siècle et demi sur le possible point de rupture entre les deux, et sur la compatibilité des deux systèmes.

3 De ce point de vue, il ne faudrait pas parler d'économie de marché socialiste, mais bien d'économie de marché d'État. Depuis ces vingt dernières années, chacun attendait des transformations économiques en cours une fragmentation et un dépérissement de l'État, sans trop savoir ce qu'on allait mettre à la place. Il me semble qu'il y a eu, au contraire, reprise et consolidation de l'État dans de nombreux domaines : consolidation technocratique, consolidation des règles, puissance renforcée de l'État à ses différents niveaux. C'est une source de surprise et d'interrogations. Certes, cet État s'est fortement diversifié régionalement, mais nous n'avons pas assisté au passage attendu au libéralisme économique de marché, car ce marché est aujourd'hui complètement encadré, piloté par l'État. Même ses aspects anarchiques sont parfois repris en main assez fortement. Ce n'est pas là une complète innovation : des générations d'historiens ont travaillé sur l'économie mixte en Chine, et sur la place relative de -l'état et des marchands... La force du socialisme aujourd'hui, s'il existe en Chine, c'est d'avoir retrouvé cette tradition étatique et de s'être abrité derrière elle. C'est ce qui distingue le pays des autres États postsocialistes, tout au moins européens.

4 Jean-Luc Domenach : Je développerai deux points, en prenant, si l'on peut dire, l'économie «par les bords ». Le premier point porte sur l'importance psychologique et politique décisive de la croissance, et plus encore de l'espoir de la croissance. Ce régime ne tient que par des réussites qui donnent à croire qu'il y aura encore mieux plus tard, car les résultats acquis ne peuvent suffire en eux-mêmes. Les Chinois sont souvent mécontents, mais ils imaginent qu'ils pourront acheter un réfrigérateur demain, ou une voiture dans quelques années. Voilà l'assise principale de ce régime: l'espoir qu'il donne dans l'avenir. Espoir en partie faux à mon sens, du reste, ou que beaucoup d'économistes considèrent comme tel, puisque l'inévitabilité de la montée des coûts qui accompagnent le développement économique chinois devient une évidence: coûts sociaux, coûts d'équipement, coûts de recherche...

5 TC : N'est-ce pas dû en particulier aux privatisations qui se mettent en place, par exemple aux transformations du droit au logement, à l'apparition de systèmes éducatifs privés, analysés dans ce numéro. Qu'en est-il par ailleurs de la santé ? La nouvelle politique économique a-t-elle, au total, un coût social élevé ?

6 J.-L. D. : Effectivement. Ce qui était auparavant considéré comme des coûts nécessaires de la réforme est désormais perçu comme de vrais scandales indignes d'un pays moderne. Au début du processus, Deng Xiaoping a réussi à convaincre la Chine entière qu'il fallait que l'État se décharge de toute une série de ses fonctions. Désormais, les progrès de l'économie ayant suscité dans la population des exigences nouvelles, les attentes s'aiguisent en matière d'éducation et surtout de santé, les lacunes de la protection sociale posant un énorme problème. Tout cela va coûter infiniment cher. 
7 Mon deuxième point porte sur la nature de l'État. Cet État qui dirige ou qui contrôle tout n'est pas seulement une machine politico-administrative, c'est une couche sociale, l'équivalent chinois de l'élite à la fois bureaucratique et entrepreneuriale qui a industrialisé les sociétés occidentales au fil du XIX· siècle. Le spectacle de l'État met en évidence cette couche sociale qui se répartit les rôles. Les plus -puissants, ceux qui comptent vraiment, tiennent le pouvoir politique. Leur parentèle, leurs frères, leurs cousins siègent dans les directions des entreprises d'État ou des -entreprises privées. Les contradictions qui auraient pu se développer si ces dirigeants avaient appartenu à des milieux sociaux différents se résolvent parce que ce sont finalement les mêmes familles en jeu, les mêmes intérêts, et en dernière instance la même prudence généralisée. Cette prudence généralisée est une des clés de la Chine d'aujourd'hui, dont la logique repose sur ce raisonnement : si l'on veut continuer à bénéficier du système et à le contrôler, il faut serrer le jeu. Il faut commencer à faire des politiques sociales, des politiques d'équipement... Jouons serré, jouons sérieux, jouons politique. D'où cet aspect très cybernétique du pouvoir politique.

8 F. G. : La volonté affichée du gouvernement chinois aujourd'hui, c'est de rompre avec le modèle de croissance des années Deng Xiaoping, une croissance débridée, extensive, tirant un chèque sur l'environnement, sur la situation démographique très favorable, sur la libéralisation tenant lieu de politique sociale. Ce modèle transitoire a tenu bon un certain temps, mais chacun sait qu'il ne peut plus durer, car son coût est devenu trop élevé. Au fond, l'économie chinoise a fonctionné comme le fit l'économie américaine pendant la marche vers l'Ouest : pendant vingt ou vingt-cinq ans, plus on avançait, plus les coûts baissaient, grâce entre autres aux effets de la jeunesse et de la mobilité de la population, un facteur essentiel au cours des vingt dernières années. Mais on finit inévitablement par buter sur des logiques de coûts. Depuis deux ou trois ans, on parle de l'élévation du coût de la main-d'œuvre dans un certain nombre de secteurs, et aussi de pénuries localisées de main-d'œuvre. Mais on pourrait parler aussi du coût d'entrée qui subitement s'élève dans l'immobilier, un secteur par lequel s'est largement constituée la classe ou la caste actionnaire dont parle Jean-Luc Domenach, un secteur d'une importance sociale gigantesque dans la Chine d'aujourd'hui.

TC : En raison de l'hypercroisssance des mégalopoles?

F. G.: Oui. Sur tous ces plans, il apparaît que le mécanisme de l'ère Deng Xiaoping n'était pas indéfiniment reproductible. Pensons au phénomène générationnel mis en lumière par Thomas Piketty à propos du développement des inégalités dans la société française depuis 1945. Une remise à niveau s'est produite après la guerre, mais une ou deux générations après, les inégalités se sont accumulées au sein d'un système de marché. En Chine, la tabula rasa, le moment zéro, ce fut la fin du maoïsme. Aujourd'hui, tous les mécanismes inégalitaires sont en mouvement.

11 TC : C'est dire que la courbe de la très forte croissance de 9 ou $10 \%$ l'an masque des déséquilibres dont les prix vont devoir être payés?

12 F. G. : En effet. La courbe n'est pas continue et s'accompagne d'inégalités désormais galopantes.

13 J.-L. D.: D'où des dépenses croissantes pour élever des contre-feux face à ces inégalités.

TC : Outre les inégalités sociales, on observe aussi des disparités régionales. Peut-on dire que le système économique mis en œuvre il y a plus d'une -génération a dessiné 
une nouvelle géographie économique chinoise? Ou n'a-t-il fait que cristalliser une opposition structurelle entre les provinces côtières - voire les dix-huit provinces - et tout le reste, les périphéries lointaines du Tibet ou du Xinjiang, mais aussi les périphéries plus proches comme le Yunnan ou la Mandchourie?

F. G. : Naguère, la chine était fragmentée par la distance, par l'insuffisance de transports et par des structures administratives aussi étanches que bien des États. Pour la seconde fois dans l'histoire de la Chine (car l'Empire avait réalisé une première forme d'unité avec les grands travaux hydrauliques), une politique de dépenses publiques systématiques en matière d'infrastructures de transport a vu le jour. Ajouté à la construction de dizaine de milliers de kilomètres d'autoroutes et au développement des lignes aériennes et ferroviaires, le droit donné aux gens de quitter la terre est une véritable révolution sociale.

TC : Quand commence ce grand tournant?

F. G.: Sans doute fin 2002, quand les paysans ont été autorisés à vendre les droits d'exploitation de leurs terres. Les effets économiques de cette réforme sont très récents, mais la migration de masse existait déjà dans les années 1980, quand on comptait selon les sources entre 80 et 130 millions de migrants. De ce point de vue, le modèle économique chinois a anéanti les cadres géographiques et administratifs dans lesquels le pays avait longtemps fonctionné. C'est un phénomène de masse, sur le plan économique comme sur le plan social; un bouleversement très violent qui peut engendrer des conflits aigus. En outre les poches de pauvreté n'étaient pas limitées à l'Ouest. Elles se dispersent à travers tout le territoire, " en taches de léopard » comme disent les Chinois, y compris assez près de régions très développées.

. . 'insiste pour ma part sur ce que j'appelle la «Chine tragique ». C'est la Chine du noir de cette peau de léopard, en particulier toute la Chine centrale. En gros la Chine qui descend du Shanxi, où la sécheresse pose un problème d'irrigation dramatique, la Chine du Henan, de l'Anhui du nord et du Hubei surpeuplés. C'est une Chine qui est écologiquement, économiquement et socialement en danger. Cette Chine pose de graves problèmes, plus sérieux que ceux des zones périphériques dont on a beaucoup exagéré les difficultés, alors qu'on trouve sur les frontières des zones qui fonctionnent bien. C'est dans cette Chine en péril que les sectes protestantes prolifèrent. Les estimations qui leur donnent 200 millions de fidèles sont probablement exagérées, mais le phénomène n'en est pas moins extraordinaire. C'est cette Chine, celle où l'on part en masse travailler dans les villes, qui est saisie le plus violemment par les inégalités. Les dix années à venir seront essentielles à cet égard, car c'est dans cette décennie que le problème de ces régions sera ou non résolu, ici par l'immigration, là par l'irrigation...

19 TC : Que peut-on dire dans ce contexte du parcours démographique -chinois? Le très faible taux de croissance démographique actuel, 0,6 \% l'an, est-il perçu comme un atout permettant de redistribuer une part des fruits de la -croissance à une population presque stabilisée ? Le vieillissement de la population est-il au contraire une source d'inquiétude, à l'heure où certains analystes considèrent que l'Inde jouira d'un avantage comparatif sur la Chine, dans quinze ou vingt ans, quand elle disposera d'une masse plus importante de jeunes actifs?

20 F. G. : En une décennie, la teneur des débats chinois sur cette question s'est inversée. La politique démographique traditionnelle veillait à freiner la natalité. On s'interroge aujourd'hui, à l'inverse, sur les effets du vieillissement de la population, et sur le malaise social qu'il pourrait engendrer. On entend dire parfois que le gouvernement 
chinois lèverait le pied sur la politique de restriction de la natalité. En fait, il applique moins sévèrement cette politique, il réduit les campagnes de masse sur ce sujet, sans qu'on puisse parler d'arrêt. La situation actuelle n'est d'ailleurs pas sans avantage. D'une part, le coût de l'éducation va baisser tandis que, d'autre part, les nouveaux actifs sont plus qualifiés que les générations qui se retirent. C'est l'une des explications de la hausse surprenante de la productivité chinoise. Sur le court terme économique, la tendance n'est donc pas catastrophique. À long terme, l'optique prudentielle prévaut pourtant: une optique à l'européenne en quelque sorte, face au trou noir d'une génération de sortie du socialisme qui a confondu le fait de préparer l'avenir avec le fait d'épargner dans une tirelire. Épargne massive, épargne perdue, épargne gaspillée, entre autres par le système financier d'État à qui elle a servi de recours, ou au mieux placée dans l'immobilier, mais sans véritable mécanisme prudentiel collectif. Seulement $17 \%$ de la population est couverte par un système social public. Les détournements de fonds de retraite sont légion, comme l'illustre un scandale récent au plus haut niveau à Shanghai.

TC : Épargne perdue parce que non investie dans des opérations industrielles ou productives?

F. G. : Épargne perdue parce que pendant quinze ans l'inflation était assez forte, avant le coup d'arrêt de 1997, et parce que les placements bancaires étaient non attractifs en raison de taux d'intérêts ridicules. À cet égard, la génération de la sortie du socialisme a été spoliée. Émerge ensuite, de façon remarquable, un système prudentiel composite, où diverses formules existent. Il est de tendance -bismarckienne, puisqu'il associe la logique de la capitalisation à la logique de l'assurance par répartition. Avec ce côté très chinois de faire des expériences régionales, de privilégier les villes, et donc d'être ouvertement inégalitaire avant d'élargir le système aux dimensions du pays. Il existe aujourd'hui une caisse centrale de la sécurité sociale en Chine dont on parlera dans quelques années comme étant un des premiers investisseurs mondiaux. On ne peut donc pas dire que les responsables soient inactifs ou indifférents.

TC: C'est une réponse structurelle, discrète mais essentielle, guidée par le pragmatisme?

F. G. : C'est l'aboutissement de dix ou quinze ans de tâtonnements en tout cas plus pragmatiques que dogmatiques. Mais comme nous sommes en Chine, l'idéologie a aussi sa place : aujourd'hui, le Parti cite le modèle social d'Europe du Nord, pourtant hors de portée. Au total, dire que la croissance chinoise va être brisée parce que la population va vieillir me parait exagéré.

J.-L. D. : Il faut souligner ici les effets sociaux, déjà perceptibles ou à venir, du vieillissement de la population et de la fragmentation progressive des familles. Les classes d'âge qui vieillissent sont de plus en plus abandonnées par les jeunes générations, elles vieillissent seules. Cette population âgée va demander des bénéfices sociaux, des loisirs. Elle va chercher à consommer. Elle va donner un tour plus individuel à la vie de quartier. Ainsi apparaît une Chine très différente de celle que l'on a connue, une Chine beaucoup moins collective, beaucoup moins travailleuse, plus voyageuse, voulant profiter un peu de l'avenir et porteuse d'une autre vision de la société, une Chine aussi différente de la Chine puissante qu'admirent certains, que de la Chine noire du capitalisme débridé que haïssent les autres.

F. G. : On retrouve là quelque chose qui évoque la génération des "tempes argentées » à la japonaise, à ceci près qu'au Japon cette génération dispose d'un épais matelas 
d'épargne qui a servi d'amortisseur à la crise. Il n'est pas certain qu'en Chine cette génération vieillissante puisse préserver ses chances économiques. quantitativement et qualitativement. Ce mouvement massif aboutit en effet à renforcer l'urbanisation sans troubles sociaux majeurs, et à accélérer la formation des migrants, à commencer par celle des jeunes filles. Ces migrants se sont fait et se font aujourd'hui admettre par les autres citadins à une vitesse extraordinairement rapide. Le plus optimiste des observateurs n'aurait pas osé l'espérer. On nous avait prédit que ces migrations massives déclencheraient des révoltes épouvantables; puis qu'il n'y aurait ni révolte ni intégration; puis que l'intégration se ferait tant bien que mal, sans aide de l'État. On s'aperçoit aujourd'hui que l'État intervient, qu'il donne des soutiens, qu'il subventionne des écoles... Évidemment, tout cela se passe dans un cadre général de terrible exploitation de cette main-d'œuvre, exploitation que tout le monde accepte, y compris, et on a du mal à le croire, les premiers concernés eux-mêmes, pour lesquels le pire serait de rentrer au pays, où il n'y a pas de travail. Cette main-d'œuvre se défend d'ailleurs à sa façon, un peu comme aux États-Unis : elle se déplace en fonction des salaires qu'elle peut trouver. D'un côté, l'exploitation de la main-d'œuvre, perçue comme le ticket d'entrée pour le droit à une vie normale, pose un problème humain et moral majeur. De l'autre, du point de vue de la dynamique globale du pays, l'intégration massive des migrants à la ville apparait comme un processus tout à fait extraordinaire et assez réussi.

TC: Nous ne serions donc plus dans le contexte dépeint voici une quinzaine d'années, quand les migrants arrivant sans permis ne pouvaient se loger, ne pouvaient travailler légalement, et devenaient une manière de citoyens de seconde classe, parce que la grande transformation n'avait pas été pensée pour eux ?

D. : Quand les migrants arrivent, ils se font toujours exploiter et ils ont toujours les mêmes difficultés à se loger, mais ils trouvent du travail, finissent par se loger, et progressivement, s'urbanisent. Tout cela fera d'excellents citadins à condition que le reste marche. Le phénomène n'est plus dangereux, comme beaucoup l'ont cru autrefois. Mais si, pour telle ou telle raison, la conjoncture économique ne suivait pas, les problèmes pourraient sérieusement s'aiguiser. ce sont aujourd'hui, à la lumière de cette grande transformation économique et sociale, les structures du pouvoir. Que peut-on dire du Parti communiste chinois? Quel est le profil socioprofessionnel des dirigeants? Vous évoquiez tout à l'heure les familles dirigeantes. Les ingénieurs ou les technocrate ont-ils pris en main le Parti? Un équilibre s'est-il instauré entre les pragmatiques et les idéologues, tous cherchant à préserver leur pouvoir? Et quelle est aujourd'hui la place de l'armée dans la structure de l'État? différentes, parce que deux choses sont également vraies. D'une part, le régime reste communiste, centralisé, dominateur - fût-ce inégalement dominateur. D'autre part, le Parti et le régime évoluent lentement mais incontestablement. Si l'on s'adresse à des hommes d'affaires, il faut leur rappeler qu'il existe un comité du Parti dans l'entreprise dans laquelle ils investissent en Chine, que leur téléphone est surveillé, et que la protection des secrets technologiques et industriels devrait être pour eux un sujet 
permanent d'inquiétude. À l'inverse, face aux défenseurs des dissidents, il faut rappeler que la population chinoise n'aime pas les perdants, et qu'elle a complètement laissé tomber les dissidents, qui ne constituent plus qu'un ensemble d'intellectuels en demisolde. Aujourd'hui, le vrai mouvement intellectuel est bien souvent aux marges du Parti, voire à l'intérieur du Parti. Il se divise en gros en deux tendances. L'une est plutôt nationaliste tout en sauvegardant une petite part du maoïsme et du communisme. L'autre plaide pour une démocratie de convenance qui est aussi une démocratie de marché. Tout cela est passablement ambigu: vous entendez des cadres importants et des intellectuels reconnus assurer qu'ils veulent aller vers la démocratie, y compris par des élections libres, mais en créant une situation telle que le PCC gagne toujours. Soulignons ici que dans les élections locales, que tout le monde ignore bien qu'elles soient à peu près libres, le candidat du Parti est battu deux fois sur trois, mais deux fois sur trois les vainqueurs intègrent le Parti. Nous sommes donc bien, typiquement, dans une situation de transition.

Quant au Parti, au centre du système, rien n'a beaucoup changé. Les dirigeants habitent toujours au même endroit secret, proche du Palais impérial. Les mêmes cancans opposent toujours les uns aux autres, comme sous Mao: histoires de voisinage compliquées entre bureaucrates, qui n'empêchent pas de conclure des accords. S'y sont ajoutées toutefois diverses affiliations disons corruptrices, que l'on connait assez mal. En revanche, le reste du Parti ressemble de plus en plus à un parti d'élites. Un universitaire me disait récemment que ses meilleurs étudiants, les plus enthousiastes pour aider les pauvres ou pour agir en faveur du tiers-monde, ceux qui veulent faire quelque chose de leur vie, entrent naturellement au Parti tout en étant souvent, idéologiquement, à des années lumières du Parti. L'un des très grands professeurs de l'université de Pékin, immense spécialiste de la Révolution française et des révolutions dans le monde, est un partisan acharné de Tocqueville. Il est membre du Parti... Autrement dit, on trouve partout des gens qui, tout en étant au Parti, font leur métier aussi honnêtement ou aussi malhonnêtement que sous nos latitudes, et qui ressemblent de plus en plus aux bureaucrates et aux élites que nous connaissons.

Dernier point, qui sont ces dirigeants chinois? Je dirai qu'ils sont surtout ce que leur couche sociale leur demande d'être: des défenseurs presque anonymes d'une classe dirigeante. Les personnalités sont beaucoup moins affirmées qu'autrefois, les lignes politiques sur lesquelles elles s'affrontent sont de moins en moins spécifiées. Il se constitue une manière de directoire presque anonyme au service d'intérêts très généraux, ceux d'une couche sociale qui veut à la fois continuer à profiter de sa position tout en comprenant, comme la bourgeoisie française du Second Empire, qu'il va falloir maintenant jouer serré et faire attention pour que le système dure. C'est ce qui rend compte de la dualité de ces dirigeants qui sont à la fois des -communistes et des ingénieurs, des gens qui ressassent un vocabulaire suranné tout en conduisant des politiques souvent extrêmement fines. Ce moule produit des dirigeants comme le Premier ministre Wen Jiabao ou le président Hu Jintao, à la fois couleur de muraille et capables de définir des politiques adaptées aux besoins du moment. Jean Louis Rocca l'a dit le premier : nous avons là le premier régime communiste à avoir fait des politiques sociales.

F. G. : Effectivement, ce n'est pas un régime autoritaire classique, car il a été marqué dans sa légitimité et dans son système de contrôle et de mobilisation par l'ingénierie sociale, de façon inattendue, en dépit du déclin du maoïsme à l'intérieur du 
communisme chinois. Nous ne sommes donc pas dans une situation postfranquiste, comme on l'a cru un moment. Nous sommes encore dans une situation où le régime peut casser des systèmes, transformer des conditions politiques ou sociales. Bref, ce régime peut agir avec une cohésion interne plus forte aujourd'hui qu'au lendemain de 1989.

Deuxième aspect très important: la question de l'éthique du système. Le groupe dirigeant n'est pas seulement fait des ingénieurs du pétrole et de l'électricité issus du socialisme des années 1950 ou 1960, ou de représentants d'un nouveau capitalisme hybride, sorte de société en commandite à la chinoise. Nous avons aussi aujourd'hui des ingénieurs de l'âme, d'anciens jeunes communistes qui ont pratiqué l'ingénierie sociale quand ils ont subi le sort des cadres pendant l'ère maoïste : ils ont été envoyés dans des régions pauvres et y ont paradoxalement conservé une vision communiste sur le plan social, tout en étant méfiants vis-à-vis de la bureaucratie économique planifiée. Au total, nombre de cadres chinois sont individualistes, occidentalisés et restent curieux de tout, ont des familles qui s'enrichissent et dont les membres voyagent en Occident, d'où ils ramènent des idées et des informations. Ce groupe n'est donc pas du tout fermé, c'est là un point extrêmement important.

Troisième remarque : par légitimation sinon communiste du moins jacobine, Hu Jintao et son équipe énoncent des thèmes moraux, des thèmes anti-corruption, et des thèmes critiques des autorités locales. Ils n'hésitent pas à « lâcher » ces dernières dans certains cas. C'est aussi une soupape de sécurité face au mécontentement sur la corruption, aux émotions populaires, et cela permet aux dirigeants de garder une marge d'intervention sur l'économie ou la société. Ce n'est pas pour autant un modèle proche de celui du Parti révolutionnaire institutionnel mexicain. Hu Jintao reprend assez curieusement le mécanisme des campagnes de masse pour renforcer le contrôle collectif du Parti dans un certain nombre de domaines. C'est en ce sens qu'on parle aujourd'hui de consolidation de l'autorité, mais cette évolution joue son rôle du point de vue de l'ingénierie sociale.

TC : Jusqu'à quel point cette soupape de sécurité fonctionne-t-elle?

F. G. : Elle fonctionne dans la mesure où ce discours redonne une direction à la politique du pays, moins idéologique - il n'y a pas de communisme dans le système que morale. Les historiens de la révolution chinoise ont souligné que ce n'était pas l'idéologie communiste qui l'avait emporté par elle-même, mais bien le sens de la protestation morale qui avait provoqué le ralliement au mouvement. De ce point de vue, la rupture n'est pas encore achevée entre ceux qui ont fondé la révolution et ceux qui la gèrent aujourd'hui dans une direction imprévue. On a vu récemment le commandant de la marine mis en difficulté après des révélations sur son comportement, son train de vie et sa maitresse, preuve que le Parti a quelques instruments de contrôle sur ce qui est pourtant la caste professionnelle la plus vigoureuse et indiscutablement le lobby le plus encombrant au sein de l'appareil d'État. La chute récente du numéro un du Parti à Shanghai est un exemple d'une plus grande portée encore, tout comme la conduite, à l'été 2006, d'une campagne d'épuration du Parti qui a demandé des comptes à chacun de ses 78 millions de membres.

TC : Encombrant, le lobby miliaire, parce qu'il a trop de pouvoir par rapport aux civils, ou encombrant parce qu'il définit une ligne stratégique qui est objet de débats? aux dirigeants, l'armée réclame des crédits, tout simplement. Il ne s'agit pas seulement 
de restaurer la puissance militaire chinoise pour la projeter éventuellement à l'extérieur, mais aussi, à l'évidence, d'équilibrer, à l'intérieur, des rapports de force politiques.

TC : Voulez-vous dire que dans le rapport entre le Parti et l'armée, l'armée dispose d'une marge de manœuvre ? Ou faut-il penser que la montée en puissance militaire de la Chine est voulue au premier chef par le pouvoir civil, au terme d'une lecture des rapports de force mondiaux et d'une analyse des corrélations entre montée en puissance économique, impératif de la sécurité nationale et quête d'un statut de grande puissance émergente?

F. G. : La vision géopolitique et géostratégique des dirigeants chinois n'est pas celle des tenants de la théorie libérale des relations internationales. Elle est héritée du réalisme géopolitique du XIX. siècle. Avec en sus le souci d'éviter le piège dans lequel s'est perdue l'URSS : l'impasse de la compétition avec les États-Unis. Les stratèges chinois évoquent régulièrement cette question, et ils en ont retenu la leçon. Mais il est vrai qu'à différents moments, l'armée chinoise a été au premier plan, que ce soit au moment de la courte guerre contre le Vietnam en mars 1979, que ce soit juste après Tiananmen quand il était impérieux de réduire à tout prix les fractures politiques, ou quand il a fallu, avec l'effondrement de l'idéologie communiste, conforter une légitimation nationaliste dans les années 1990.

TC : La place de l'armée tient-elle au pouvoir qu'elle a de porter le nationalisme chinois?

F. G. : Non. Plus simplement, ne pas soigner l'armée ouvrirait une crise de légitimité qui affecterait la nation chinoise en général. Je ne dis pas que l'armée influence les politiques civiles, je dis que l'armée est très bien placée pour réclamer son dû, et pour le définir de la manière la plus large.

J.-L. D. : Les gens ne défilent pas dans la rue en disant: «l'armée avec nous!" Ils pensent du bien de l'armée parce que lorsque l'on y sert, on y apprend un métier, on y bénéficie de soldes augmentées : il y a de bonnes places dans l'armée. Mais par ailleurs, l'armée, ce sont aussi les garnisons lointaines, où les femmes d'officiers ne veulent pas aller vivre... L'armée, vue sous cet angle, est comme une administration qui a des avantages et des inconvénients, qui est utile mais sans plus. Elle n'est pas un sujet de discussion récurrent comme la corruption, Taiwan ou le Japon.

TC : Vous avez à plusieurs reprises évoqué la question de l'administration locale. Peuton en souligner ici les enjeux?

J.-L. D. : La question des appareils locaux et des dirigeants locaux est en effet essentielle, car elle est porteuse d'une très grave menace pour l'avenir. Considérons en effet l'État chinois comme un appareil administratif et politique qui couvre horizontalement l'ensemble du territoire, et qui contrôle verticalement la hiérarchie des subdivisions territoriales et administratives. Le gouvernement impose à cet appareil de mettre en œuvre une politique assez exigeante, qui consiste à la fois à réussir le développement économique (tout en s'enrichissant) et à appliquer un certain nombre de réformes, certaines cosmétiques, d'autres effectives. Ce qui est demandé aux autorités locales dans ce contexte est souvent délicat pour des dirigeants qui entendent souvent profiter au maximum de leur situation. Les divergences s'accroissent dès lors entre les fonctionnaires centraux - du moins ceux qui font leur métier purement et simplement et qui entendent que partout ce métier soit fait - et les dirigeants locaux 
dont l'avidité est extraordinaire. Un exemple: plus de $95 \%$ des membres de l'administration charbonnière du Shanxi, censée améliorer les conditions de travail dans des mines connues pour leur très grand nombre d'accidents, étaient en même temps à titre privé actionnaires de mines illégales. On a connu d'autres affaires du même ordre, comme celle de l'empoisonnement du fleuve du Heilongjiang, où depuis longtemps les dirigeants concernés étaient sous le feu de la Commission nationale de protection de l'environnement. Cette commission avait perdu la partie, jusqu'à ce que l'explosion de l'usine pétrochimique de Jilin et le rejet des polluants dans le fleuve tournent au scandale, début 2006... Depuis, les comptes ont été réglés, et les administrations provinciales épurées.

Ce laxisme intéressé qu'on vient d'évoquer dans les milieux miniers ou dans la nonprotection de l'environnement se manifeste dans tous les domaines, dans le secteur bancaire par exemple. Alors que l'État central commence à épurer les créances douteuses qui affaiblissent le système financier, on continue, malgré les contrôles établis, de consentir des prêts dans des conditions tout à fait anormales à des entreprises d'État provinciales, socialement liées aux appareils administratifs provinciaux. De telles contradictions restent supportables quand l'économie nationale connaît des taux de croissance de $10 \%$ l'an. Mais si la croissance tombait à 5 ou $6 \%$, il en serait autrement, et l'on pourrait connaître de véritables soulèvements locaux, dans les chefs-lieux de districts, de régions administratives ou même de provinces, où l'on entend parfois des choses hallucinantes, qui nourrissent la haine envers les responsables aux commandes.

50 TC: Nous avons dit tout à l'heure que le Mouvement pour la démocratie, lié aux évènements de 1989, n'existe plus. Qu'en est-il d'autres mouvements qui cherchent à conquérir un espace démocratique?

51 F. G. : En tant qu'organisation, le Mouvement pour la démocratie est bien mort comme tous les mouvements qui ont dû émigrer, entre autres parce que ses meilleurs militants ont pu ou dû quitter le pays. D'une façon plus générale, la question appelle à souligner la stratégie politique du discours d'État. Ce régime a en effet une grande maîtrise de la propagande et de l'orientation de la communication. Il a rénové les médias modernes... TC : Tout en les contrôlant...

53 F. G. : En les contrôlant, à l'image de la métaphore sur «l'économie socialiste de marché ». Internet existe, les forums sont très nombreux. Mais derrière l'apparence d'un certain degré de liberté, le contrôle est extrêmement fort à tous points de vue, y compris par identification et fichage des individus. Il en est de même pour les mass médias. Les Beijing wanbao, Nanfang ribao et autres quotidiens à grand tirage sont assez bons, assez précis et assez ouverts sur le monde. Mais à leur façon plus tranquille, ils véhiculent le discours dominant tout autant que le Quotidien du peuple. Quant à la chaîne de télévision CCTV 4, elle donne l'impression d'un monde en flammes dans lequel les Chinois sont des victimes potentielles de sauvages sur différents continents, un monde où la dangereuse puissance américaine se déploie sans retenue. Et les médias chinois comme la sociologie politique officielle insistent beaucoup sur l'existence d'un "courant de pensée dominant» (sixiang zhuliu). C'est le mainstream thought à l'américaine dans la forme, mais parfois du maoïsme à peine rénové dans le fond...

TC : Prévaut donc la volonté de s'inscrire dans la pensée dominante... 
existe un marché de la spiritualité que remarque tout voyageur passant sans arrêt, à Tokyo ou à Taipei, devant le siège de telle association d'église ou de telle petite secte d'inspiration bouddhiste. Le fait n'est pas propre à la Chine. Ce n'est pas pour autant que ces associations spirituelles seront porteuses de grands bouleversements: elles servent au contraire à supporter l'existant. En revanche, la montée des aspirations individuelles évoquées plus haut pèsera sans doute plus lourd. Que les églises, les sectes ou les cultes au sens large posent en eux-mêmes des problèmes à l'État, c'est certain. Mais cela n'implique pas qu'ils deviennent des vecteurs politiques. Rien n'annonce un phénomène à la polonaise.

TC : Les références postconfucéennes l'emporteraient donc sur l'interprétation qui verrait des ébauches de mouvements sociaux se manifester en dehors d'un espace politique totalement verrouillé?

61 J.-L. D. : Si le politique reste dominateur, il l'est moins qu'autrefois, et il libère un peu plus d'espace au social. En d'autres termes, on peut discuter de tout entre amis, si l'on ne forme pas un parti politique, et si l'on ne retrouve pas trop souvent les mêmes personnes discutant sur les mêmes thèmes sensibles.

J.-L. D. : Et pas sur Internet. Il convient par ailleurs de mesurer tout le poids de la professionnalisation de la société, et donc du rôle de ceux qui -entendent bien faire leur métier, qu'il s'agisse de réparer les voitures, de réparer les hommes, ou de réparer les injustices. Médecins, avocats, juges, ou autres métiers commencent à constituer des corps, la professionnalisation évoluant donc vers une «corporatisation» des élites. On pense au XVIII siècle et à ce que disait Voltaire aux académies provinciales : « Messieurs, formez des corps!» Le Parti sait tout de ces corps, qui ont des objectifs différents des siens. Dans une affaire judiciaire par exemple, le comité du Parti attaché au tribunal exprimera une position inscrite dans la continuité des pratiques. Mais il se heurtera éventuellement aux juges qui, vecteurs de changement, lui diront : « Désolés, mais nous ne pouvons pas défendre cette interprétation des textes, à moins que vous ne nous en apportiez une justification légale.» La discussion se passe dès lors sur le terrain professionnel. Tout aussi porteur d'avenir est l'idéal professionnel de jeunes 
journalistes, à la fois nombreux et idéalistes, qui exercent une pression de tous les jours pour une information meilleure.

TC : On a souvent entendu dire que la contradiction entre libéralisation économique et verrouillage politique serait insoluble. Vous montrez plutôt que le pouvoir chinois trouve en continu des moyens de gérer la dialectique entre -l'ouverture de l'économie vers le monde et la persistance d'un système politique très contrôlé ?

F. G. : Il faut voir les choses dans la durée. Le pouvoir n'a pas été réformé : il a été déformé, modifié par les interactions avec la société internationale et par les pressions qui se sont exercées sur lui. Il serait faux de penser qu'il existerait un bloc de mandarins conservateurs attachés à cinq mille ans d'histoire bureaucratique, qui ferait face à une économie globale déchaînée, tout en sachant de temps en temps la canaliser. Trois étapes ont marqué l'évolution de la Chine sur ce plan. On a un peu oublié la phase des droits de l'homme depuis que l'Europe a décidé en 1997 de ne plus mettre cette question au premier plan. Dans cette phase, le pouvoir fut contraint de répondre constamment à des interpellations, formulées de façon collective notamment à la session annuelle de la Commission des droits de l'homme de l'ONU à Genève, ou énoncées de façon bilatérale, particulièrement par les dirigeants américains. Dans le même temps, ce même pouvoir mettait un point d'honneur à dénier toute influence internationale sur ce sujet : tel était le leitmotiv de Deng Xiaoping. Quoi qu'on pense du degré de répression qui existait alors, il était bien plus faible que celui exercé par la génération précédente, ou lors de l'épisode de Tiananmen en 1989. C'est parallèlement à cette répression politique amoindrie que l'essor indéniable de la légalité s'est opéré, véritable phénomène de société. La mise en place d'élections locales villageoises jamais étendues depuis - date aussi de cette époque-là.

TC : C'est le début des années 1990 ?

F. G. : Oui. Vient ensuite la phase "Organisation mondiale du commerce ", à la fin des années 1990. Zhu Rongji, alors Premier ministre, use de la question de l'entrée de la Chine à l'OMC comme d'un aiguillon pour accélérer la réforme intérieure de l'économie chinoise. De fait, l'homogénéisation croissante du droit commercial chinois avec le droit international et l'extension de la liberté de concurrence (même si des difficultés particulières attendent les entreprises étrangères) modifient durablement la structure de l'économie chinoise, aussi bien que ses règles de fonctionnement.

La troisième phase est toujours en cours. Elle correspond à la demande des partenaires qui pressent la Chine d'assumer ses responsabilités internationales. Pékin y répond parfois positivement, parfois négativement. Mais la Chine sent bien qu'elle ne peut pas continuer son expansion commerciale internationale sans offrir quelque chose de positif à la communauté internationale en retour. Lui demander de voter à l'ONU contre le gouvernement de Khartoum sur le Darfour était peut-être sans espoir, vu les intérêts économiques chinois au Soudan. Il s'agit plutôt de l'encourager à jouer un rôle différent dans l'environnement régional. Apparaissent ainsi les thèmes de la responsabilité et du soft power, par exemple à propos de la Corée du Nord. Les dirigeants chinois risquent de devoir accepter des formes d'intégration internationale un peu plus fortes que ce qu'ils avaient envisagé.

69 TC : Le premier cercle étant celui de la politique de voisinage ? 
70 F. G. : Oui. Rappelons ici les concessions faites par Pékin, dans le cadre de cette politique de voisinage, lors de la crise financière asiatique de 1997, qui ne fut pourtant pas sans impact sur la Chine.

71 J.-L. D. : Si l'on remet en perspective la question des droits de l'homme posée par l'Occident à la Chine et celle du commerce de ce même Occident avec la Chine, en un sens tout le monde avait raison. Ceux qui disaient qu'il fallait donner une extrême importance au commerce avec la Chine pour la conduire à s'ouvrir, avaient raison. Et ceux qui disaient qu'il ne fallait rien lâcher sur la question des droits de l'homme avaient également raison. C'est cette combinatoire qui explique le succès relatif de la dernière décennie dans ce domaine. Les gouvernements étrangers prétendant commercer tout à fait librement avec la Chine donnaient certes la priorité aux échanges, mais sans se priver d'aborder aussi la défense des droits de l'homme, souvent sur des cas individuels précis. Les Chinois ont ainsi toujours eu l'impression de trouver des partenaires qui leur ressemblaient, qui promettaient beaucoup mais sans faire tout ce qu'ils avaient promis. Tout cela a permis des avancées, à l'exemple de la coopération franco-chinoise en matière de droit et de prisons, une des rares politiques efficaces que la France ait menée dans le cadre bilatéral avec la Chine, qui, j'en témoigne, a conduit des procureurs ou des juges à mieux résister à la Sécurité publique et développer de véritables stratégies de consolidation professionnelle. Notons au passage que le nombre de détenus politiques oscille aujourd'hui suivant les sources entre 5000 et 30000 personnes (si l'on inclut les membres emprisonnés du Falungong). Il se comptait en millions dans les années 1950. La tendance est à condamner moins, et à des peines plus courtes. Si ce chiffre est ramené à l'ensemble de la population chinoise, la Chine sort des pires régimes au monde. Le pire absolu existe encore, tels les travaux forcés dans les mines de montagnes. Mais on trouve aussi en Chine des prisons mieux construites et mieux organisées que certaines prisons françaises.

TC : Dans le cadre de la dialectique entre l'externe et l'interne, peut-on considérer que «la montée en puissance pacifique » de la Chine, formule consacrée, est un ferment de sa modernisation?

73 F. G. : La dialectique interne-externe est très importante parce que la Chine connaît une période d'expansion extérieure exceptionnelle, marquée par l'essor de son commerce et par l'acquisition de technologies étrangères. Elle a retourné les règles du marché à son profit, et elle les revendique même parfois contre ses partenaires étrangers tentés par le protectionnisme. Dans le même temps, son économie intérieure peut être qualifiée au mieux de colbertiste, au pire d'un souverainisme organisé. Sur ce plan, l'intérêt de la communauté internationale réside certes d'abord dans la stabilité de la Chine et de son développement, mais il importe aussi d'amener la chine à respecter un certain nombre de règles sur le plan économique et social : droits de propriété intellectuelle, garanties sociales, réglementations salariales. Le propos paraît très européen, mais la pression majeure sur ces questions vient des États-Unis. Ces pressions extérieures sont une donnée importante, mais pour être efficaces, elles ne doivent pas devenir trop explicites.

Deuxième clé de cette dialectique, il importe d'élargir la nature des échanges -culturels et de coopération avec la Chine. Il ne suffit pas d'accueillir uniquement des ingénieurs, des techniciens, des scientifiques, ou des financiers chinois. Il faut aussi faire place aux avocats, aux policiers, aux journalistes, aux littéraires, aux chercheurs en sciences sociales. Il faut poursuivre ce qui est engagé, en sachant que l'individu chinois est 
poreux, ouvert, et qu'il a un sens assez fort des valeurs individuelles. Il est extrêmement important qu'il ait une expérience de systèmes sociaux et politiques dans lesquels l'équilibre entre l'individu et le collectif est différent de celui qu'il connaît. Si l'on veut influencer la Chine dans cette phase de transition, on ne peut fixer seulement quelques points d'arrêt. Il faut aussi formuler des propositions positives.

J.-L. D.: Dans ce rapport à l'Occident, je voudrais insister sur l'importance de l'incertitude sur l'avenir qui étreint la société chinoise. De l'expérience de l'ouverture, et de sa meilleure connaissance des pays occidentaux, elle tire souvent la conclusion qu'elle n'est finalement pas sûre de réussir, et cultive un certain sentiment d'insécurité. Nombre de Chinois ont l'impression que l'Occident est tellement fort qu'ils ne le rattraperont jamais. Je suis très frappé par le pessimisme ambiant chez les grands acteurs sociaux. Je disais à un économiste : " Vous développez votre niveau de vie, vous aussi vous aurez vos immigrés. " J'étais d'ailleurs confirmé par les faits puisqu'il existe maintenant une petite colonie africaine à Pékin. Il me répondit pourtant : «Ce n'est pas possible! Il n'y aura jamais assez d'étrangers pour vouloir venir chez nous. » Dans certains cas extrêmes, on a l'impression que se développent à la fois deux réactions contrastées : le désir de fuite et la haine de l'Occident. Et que le nationalisme chinois est en partie un nationalisme de fuyards.

TC : Haine contre l'Occident?

J.-L. D. : Haine contre les meilleurs, les Américains, qui ont atteint un niveau que la Chine n'atteindra jamais ou en tout cas n'est pas prête d'atteindre. Haine aussi contre le plus proche des meilleurs, le frère toujours premier de la classe, le Japonais. Puisqu'il est proche, on croit pouvoir le bousculer facilement. Ce nationalisme xénophobe ne demande qu'une allumette pour embraser un quartier. Le Parti communiste étouffe les affaires pourtant nombreuses, où des rassemblements se produisent très vite autour $\mathrm{du}$ moindre accident de circulation, du moindre délit, du moindre incident auxquels un étranger est mêlé. Et à l'inverse, l'attraction de l'Occident, et des États-Unis en particulier, est frappante. Beaucoup seraient prêts à émigrer aux États-Unis, même sans la garantie de pouvoir faire venir ensuite leur famille.

78 F. G. : La fascination de l'Occident joue de fait surtout par l'effet miroir des États-Unis, leur modernité, leur dynamisme, leur individualisme, leur acceptation des inégalités. Mais en même temps, la tentation nationaliste continue d'être alimentée par une relecture lacunaire de la tradition nationale.

TC : Pour conclure, que penser de la stabilité d'un régime qui paraît bouger par petites touches pragmatiques plus qu'on ne le dit souvent, face aux vecteurs d'évolution de la société chinoise?

J.-L. D. : Un point décisif, c'est que tout cela ne peut durer qu'à une condition : le taux de croissance doit demeurer très élevé. Car par ailleurs, dans la population rien n'a été oublié du passé, rien n'est oublié du présent - ce mépris pour les corrompus -, et rien n'est oublié du projet d'avenir formulé par le pouvoir. Les Chinois ne veulent pas ce que le régime prétend encore vouloir. Ils veulent une voiture, ils veulent des droits, ils veulent jouir du progrès, ils veulent qu'on les laisse en paix. Il existe une manière de " gentlemen agreement ", la population n'admettant ce régime que dans la mesure où il fournit de la croissance. Mais comment prévoir ce qui se passerait si la croissance chutait de plusieurs points? 
81 F. G. : Il semble y avoir une course de vitesse entre deux tendances : la désagrégation de l'idéologie, la montée de l'individualisme, la fracture sociale et le risque de voir un mouvement social ou même moral se dresser contre une croissance inéquitable. Mais dans l'autre sens, la transformation de la bureaucratie idéologique en une technocratie moderne, son pacte avec les élites professionnelles, grandes gagnantes en l'absence de modèle social. Et puis il existe un renouveau marqué de l'éducation, un État qui saute les étapes en matière scientifique et technologique - y compris en négociant leur transfert! - et une incroyable aptitude à capter et reprendre les modèles les plus divers. Comme Jean-Luc Domenach, je pense toutefois que le chiffre annuel de la croissance économique - et donc aujourd'hui, l'aptitude ou la volonté des grands marchés de continuer à absorber la déferlante des exportations chinoises - est le plus sûr indicateur de tendance en matière de stabilité ou d'instabilité politique.

\section{INDEX}

Mots-clés : état, évolution sociale, parti communiste, réforme

Index géographique : Chine

Keywords : China, communist party, reforms, social change, state

\section{AUTEURS}

\section{JEAN-LUC DOMENACH}

Directeur de recherche à la Fondation nationale des sciences politiques (FNSP), JeanLuc Domenach a fondé en 2002 l'Antenne franco-chinoise de sciences humaines et sociales de Pékin dont il a quitté la direction en juillet 2006. Il a dirigé le Centre d'études et de recherches internationales (CERI) (1985-1994), puis assumé la direction scientifique de la FNSP (1995-2000). Ses publications ont porté sur la politique intérieure et extérieure de la Chine populaire ainsi que sur les relations internationales en Asie orientale. Ses derniers ouvrages sont Chine : l'archipel oublié (Paris, Fayard, 1992), La Chine 1949-1994 (Seuil, 1995, en collaboration), L'Asie retrouvée (Seuil, 1997, en collaboration), L'Asie en danger (Fayard, 1998), L'Asie et nous (Desclée de Brouwer, 2001) et Où va la Chine? (Fayard, 2002).

domenach@126.com

\section{FRANÇOIS GODEMENT}

Professeur à l'Institut d'études politiques de Paris, François Godement a fondé Asia Centre, Centre études Asie en 2005. Il est aussi co-fondateur du Council for Asia-Europe Cooperation et anime le secrétariat européen du Council for Security Cooperation in the Asia-Pacific (CSCAP). Ses recherches portent sur les relations internationales et sur les questions stratégiques en Asie orientale, et sur la Chine contemporaine. Parmi ses derniers ouvrages : La renaissance de l'Asie (Odile Jacob, 1993), Communismes d'Asie : mort ou métamorphoses (Complexe, 1994, co-dirigé avec J. L. Domenach), Dragon de feu, dragon 
de papier. L'Asie a-t-elle un avenir ? (Flammarion, 1998), Chine - États-Unis, Entre méfiance et pragmatisme (La Documentation française, 2001). Il co-dirige aussi l'annuaire Asie à la Documentation française.

f.godement@centreasia.org 